\title{
MOVIMIENTOS SOCIALES PARA EL RECONOCIMIENTO DE LOS MOVIMIENTOS INDÍGENAS Y LA ECOLOGÍA POLÍTICA INDÍGENA
}

\author{
SOCIAL MOVEMENTS FOR THE RECOGNITION OF THE INDIGENOUS \\ MOVEMENTS AND THE INDIGENOUS POLITICAL ECOLOGY
}

José Guadalupe Vargas-Hernández

Institute of Urban and Regional Development. University of California at Berkeley. Correo electrónico: jvargas08@berkeley.edu.

\begin{abstract}
RESUMEN
El propósito de este trabajo, es analizar el impacto que los movimientos sociales tienen en el reconocimiento de los movimientos indígenas, y más específicamente en la ecología política indígena. El componente étnico de los movimientos indígenas, se orienta a la conclusión que no pueden ser estudiados como otros movimientos sociales, bajo un acercamiento teórico de los movimientos sociales. Finalmente se analiza la tendencia de la transnacionalización de los movimientos indígenas.

Palabras clave: Ecología política indígena, movimientos indígenas, movimientos sociales, transnacionalización de los movimientos indígenas.
\end{abstract}

\begin{abstract}
The purpose of this work is to analyze the impact that social movements have in the acknowledgement of indigenous movements, and more specifically in the indigenous political economy. The ethnic component of indigenous movement is oriented toward the conclusion that they can not be studies as the other social movements under the theoretical approach of social movements. Finally, it is analyzed the trend of transnationalization of indigenous movements.
\end{abstract}

Key words: Indigenous Movements, indigenous political economy, social movements, transnationalization of indigenous movements. 


\section{INTRODUCCIÓN}

Los movimientos sociales cortan a través de las clases sociales, lo étnico, los niveles de educación rural o urbano, etc., y a través de todos los niveles locales, regionales, estatales nacionales, internacionales y globales, formando conexiones entre el medio ambiente y el desarrollo, la salud, los derechos civiles, derechos indígenas, etc. (Brecher y Costello 1994; Carruthers 1995). Los movimientos regionales indígenas de México han sido investigados por Mejía y Sarmiento (1987), Moguer et al. (1992) y Warman y Argueta (1993), así como por los periódicos Ojarasca y Cuadernos Agrarios. Los movimientos nacionales ven el logro de la soberanía nacional como el medio para la democratizaron de las clases étnicas.

Evers (1985) lista los nuevos movimientos sociales que incluyen grupos de invasores, consejos de vecinos, comunidades eclesiales de base (CEBs) apoyados por la iglesia, asociaciones indígenas, organizaciones de mujeres, comités de derechos humanos, grupos de jóvenes, actividades artísticas y culturales populares, grupos de alfabetización, coaliciones para la defensa de las tradiciones regionales, los grupos ambientalistas, grupos de autoayuda entre desempleados y gente pobre, asociaciones de trabajadores organizados independientemente e incluso en oposición a las estructuras tradicionales de los sindicatos.

Muchos movimientos sociales abrazan un alcance más amplio de los asuntos sociales, de género, sexuales, etc., tradicionalmente defendidos por los movimientos de derechos humanos, para llegar a ser verdaderamente representativos de sus comunidades. Son una extensión del descontento de la periferia. Su éxito se basa en su habilidad para llevar la necesidad de su existencia dentro de un continuo histórico definido en el desarrollo reciproco y paralelo, de movimientos étnicos e ideológicos en competencia con el Estado.

La expansión y consolidación del activismo de los movimientos sociales, en movilización de los movimientos nacionales, con predominancia sectaria étnica nacional, determina la naturaleza de movimiento ideológico contra el Estado. Los movimientos nacionales tienen como precondición el papel expansivo del centro del estado, la creación de elites inspiradas en la polarización social y la expresión de una etnicidad reactiva. La agencia del nacionalismo es un movimiento de emancipación de grupos marginados que han sido negados el acceso a las estructuras de oportunidad política debido a su etnicidad (Tilly 1993).

\section{Naturaleza y origen de los movimientos sociales indígenas}

Los movimientos indígenas, están formados por las luchas por la identidad y la necesidad para abrir un espacio para la sobrevivencia dentro de la política nacional, y en ambiente económico y social. Los movimientos agrarios y étnicos conscientes de la lucha por la identidad, empiezan a establecer sus demandas como derechos -derechos de las tierras, o derechos educacionales. 
Los movimientos sociales indígenas, ganan acceso a la esfera política basados en la solidaridad entre los miembros y su capacidad para crear nuevas formas de relaciones sociales y prácticas como antagonismos compartidos hacia otros, en los cuales el poder no es central, definido como una continuidad en la relación del conflicto entre los actores colectivos y las autoridades (Evers,1985).

Los movimientos indígenas, están formados y dirigidos por la gente pobre y marginada, participando en una amplia variedad de arenas políticas, con demandas por el Estado plurinacional como el caso de México y el Ecuador, el cual permite hacer la transición de un movimiento social a una entidad política. El Estado crea clases con bases étnicas, dejando poca opción para ver los movimientos nacionales, como el solo agente de la movilidad socio política. Un movimiento que se moviliza busca una justificación ideológica para su organización de oposición.

El movimiento nacional, definido en términos de protección de reclamaciones de lenguaje, religión y diferencias étnicas no se sostiene (Deutsch, 1962; Connor, 1977). El movimiento nacional es una reacción al orden político definido en la predominancia étnica (Connor, 1977). El movimiento nacional es transitorio y la ideología nacionalista es una doctrina de cambio puestas en comunidades étnicas y más estáticas (Deutsch, 1963). El movimiento nacional es un agente de oposición determinada para redibujar la naturaleza de las relaciones políticas, étnicas y de clase dentro de una entidad de Estado dada.

Debido a la predominancia de ciertos grupos étnicos en regiones geográficas demarcadas, algunas naciones enfrentan movimientos que utilizan simbolismos culturales como medios de expresión de sus descontentos con y opuesto a lo que el Estado defiende (Boal y Neville 1982; Curtin et al., 1984; Watson, 1996).

El nacionalismo, es una fuerza unificadora dentro de los movimientos sociales que ofrecen un repertorio ideológico para los movimientos periféricos. Pero los movimientos profesan su propia solución étnica (Wilson y Jerry, 1996). El movimiento nacional a través de la movilización de los valores culturales étnicos, amenazan la identidad de las elites, la legitimidad del Estado y su lugar dentro de la estructura orgánica de la sociedad (Havel, 1985).

Los movimientos sociales son activos y constructivos que parten de las sociedades civiles modernas en tanto que empujan hacia nuevos valores, identidades y paradigmas culturales. La prioridad de los movimientos indígenas son los programas educacionales bilingües o recobrar las culturas tradicionales.

Los movimientos indígenas de base, desean cambios en las políticas de gobierno y desarrollan formas alternativas de manejar colectivamente las comunidades sociales. Los movimientos populares de Latino América y el mundo desarrollado son definidos por nuevos elementos: la inclusión popular de sectores dentro de la sociedad civil y su habilidad para retar al Estado, tales como el caso de los movimientos indígenas (Cardoso y Correa, 1987). 
Los movimientos indígenas son caracterizados como procesos de construcción nacional en la búsqueda de identidades colectivas, basada en la tradición cultural. Los movimientos indígenas retan a la búsqueda de equilibrio entre la identidad cultural y los logros políticos, económicos y sociales dentro de una sociedad capitalista.

Los movimientos indígenas no son homogéneos y no incluyen todos los grupos étnicos en el país, pero tiene muchas expresiones diferentes y sobre pasa diferentes entidades a niveles locales regionales y nacionales. Los movimientos locales indígenas y ambientales son pacíficos y desarmados, y se fundamentan en instrumentos legales, construyen ligas con las Organizaciones No Gubernamentales, iglesias y la comunidad científica.

El desarrollo del movimiento indígena local se incrementó con movilizaciones desde debajo de las alianzas políticas y de las elites orientadas a la reforma que controlan la política del Estado. Los movimientos sociales con frecuencia intentan ocupar las grietas en el sistema desde abajo, demandando acceso al Estado mientras que articulan sus propios intereses. Los movimientos a niveles locales están basados en su etnicidad y comunidad con limitada movilización y a nivel regional, los movimientos indígenas son asociaciones integradas de grupos étnicos. La etnicidad otorga un sentido de permanencia que los movimientos sociales ven y que son más propensos a la movilización del movimiento y resulta de la naturaleza dinámica de la interacción social étnica.

De acuerdo a Estrada (2003), las organizaciones indígenas incluyen:

Cuadro 1. Organizaciones Indígenas

\begin{tabular}{ll} 
Movimiento Nacional Indígena de la Confederación & \\
Nacional Campesina & MINI-CNC \\
Confederación Nacional de Pueblos Indígenas & CONAIN \\
Unión Nacional de Organizaciones & Regionales \\
Campesinas Autónomas & UNORCA \\
Frente Nacional de Pueblos Indígenas & FIPI \\
Consejo Nacional Indígena & CNI \\
\hline
\end{tabular}

Cuando los ciclos ideológicos se entrecruzan con las olas de movimientos de protesta social, es difícil demostrar que las altas y bajas de los movimientos sociales indígenas coinciden con un ciclo ideológico y su identidad en la historia. Los movimientos emergen de los ciclos de acción-reforma-acción más invadidos dentro de la sociedad (Tarrow, 1996; Maguire, 1996). Ninguno de los movimientos indígenas o movimientos sociales en México, tiene un apoyo ideológico por la prohibición de drogas.

Conflictos de clase y étnicos están en la base de la lucha por la identidad de los movimientos de indígenas, que tienen sus raíces durante el periodo colonial. Que los 
nuevos movimientos sociales construyen nuevas identidades enraizadas en el pasado, es un dilema que surge con respecto a los movimientos indígenas, que miran a los tiempos precoloniales para proponer una nueva forma de comunidad.

A pesar de que los movimientos indígenas tienen un componente étnico importante, comparten elementos comunes con otros movimientos sociales. Hay diferencias complejas y similitudes entre los movimientos indígenas de norteamérica y los movimientos indígenas de México.

Los movimientos populares sociales responden al tiempo de sus comunidades y la política institucional, mientras que los gobiernos usualmente difieren en su sentido de prioridades y tiempos, creando tensiones. Las comunidades indígenas y sus aliados con las comunidades no indígenas y los movimientos amplios, tienen efectos de transformación en las estructuras de gobernabilidad. El caso ecuatoriano explica la doble dinámica de los tiempos internos de un movimiento popular y el tiempo externo de la política nacional. La alianza política forjada detrás de la base de las comunidades, fue el inicio de un constante y progresivo despertar de los movimientos indígenas en el Ecuador, que en su mas alto nivel fue la referencia para todos los latinoamericanos (Zibechi, 2004).

El renacimiento étnico niega los movimientos de protesta de comunidades movilizadas (Schöpflin, 1995; Fukuyama, 1994) e ignora los movimientos sociales como una ideología de transformación política porque divide a la gente entre las líneas colectivas y minimiza lo individual dentro de los procesos políticos de representación.

\section{Movimientos sociales para el reconocimiento de los movimientos indígenas}

Movimientos sociales indígenas, masivos, radicales y anti corporativos en México pueden inspirar ideas a través de los diferentes periodos históricos. Hay muchas rebeliones y movimientos de poblaciones indígenas en defensa de sus derechos durante los tres siglos de gobierno de la colonia. Estas rebeliones y movimientos continuaron después de la independencia en 1821. En el Siglo XIX los movimientos nacionales liberados por sí mismo de las elites monárquicas hegemónicas con base étnica.

La doctrina que pone a la nación en el centro de la movilización política de oposición a través de los siglos XIX y XX se expanden como la garantía de legitimidad y liberación para ser utilizada por los movimientos en la periferia étnica (Schöpflin 1995).

Los movimientos sociales indígenas demandan derechos políticos, económicos y sociales, enraizados en el periodo colonial y post colonial, enfrentando una lucha de clases y un conflicto étnico, bajo un proyecto político identificado dentro de un contexto político nacional y la lucha por la tierra. Los campesinos y pueblos indígenas movilizados luchaban contra la privatización de sus tierras y recursos. Los movimientos indígenas sin tierra en México, llevan la reforma de la tierra hacia el centro del escenario. 
De una estructura autoritaria corporativista de los años del desarrollismo en México, emergieron los movimientos sociales e indígenas. Las organizaciones de oposición independiente y los movimientos indígenas estuvieron luchando por cambios mientras los campesinos afiliados al PRI o los pobres urbanos lograban las recompensas.

En las últimas cuatro décadas, los movimientos indígenas son movimientos sociales y ya no movimientos revolucionarios, más involucrados y organizados en Latinoamérica que durante los periodos de los cincuentas y sesentas. Los movimientos indígenas tienen preocupaciones similares a otros movimientos sociales, que buscan cambiar ya sea la sociedad en sí mismas o la posición de un grupo en la sociedad.

Desde los sesenta, hay una correlación entre el desarrollo entre la movilización cívica social inicial y la emergencia de cuestiones étnico nacionales en el desarrollo de la organización de los movimientos nacionales (Connor 1977; Nairn 1993; Hroch 1996). La estrategia de los movimientos de campesinos indígenas desde los sesentas incorpora otros componentes en la lucha, educación bilingüe intercultural, reflexión contra la estructura del Estado, análisis del sistema político, etc.

La lucha por el reconocimiento de los derechos indígenas, es uno de los más importantes movimientos sociales en México. Antes de los setentas, las organizaciones de campesinos existentes no representaron a los asuntos indígenas. Algunos movimientos locales fueron defecciones del partido corporativista que estuvo en el poder, mientras que otros emergieron de una apertura dentro de la clase política gobernante, en los inicios de los setentas y han sido promovidos independientemente desde abajo, con una fuerte movilización basada en la identidad étnica, como en el caso de Juchitlan, el pueblo mercado Zapoteca de Oaxaca.

En la década de los sesenta del siglo, pasado hubo un efluvio en el resurgimiento de la etnicidad como medio de movilización de movimientos de oposición. A nivel nacional los movimientos indígenas iniciaron en 1975 con las organizaciones indígenas, tales como el Consejo Nacional de Pueblos Indígenas (CNPI), y la Asociación Nacional de Maestros Bilingües. Desde 1975 ha habido una insurgencia de movimientos indígenas y han elevado nuevas demandas y defensa de sus valores culturales. Sin embargo, la movilización social indígena ha estado involucrada en luchas de campesinos regionales en todos los setentas y ochentas.

Los ochentas fueron tiempo de movilización social y expansión de los movimientos de campesinos e indígenas de México. La sociedad civil indígena de México en algunas regiones ricamente texturizada y delgada, fuertemente estructurada por el clientelismo en otros, refleja los legados históricos de los movimientos pasados desde abajo y las aperturas desde arriba.

Los movimientos de derechos humanos e indígenas, son una forma antigua de organización política que es revitalizada en nuevas formas de movimientos sociales. Los nuevos actores sociales tales como las mujeres, maestros, estudiantes, grupos étnicos y movimientos ambientales aparecieron, además de los movimientos laborales y de campesinos existentes, los cuales fueron reprimidos o eliminados por el Estado. Las 
gentes indígenas creen y participan en sindicatos, partidos políticos o cooperativas que, hasta 1980, no articularon sus demandas en términos de su identidad, mas bien tendieron a identificarse por si mismo como organizaciones de campesinos.

Después de 1980, los movimientos de campesinos indígenas activamente participaron en los procesos de la democratización de Latinoamérica (Horst, 1998). La renovación de los movimientos indígenas en todo México, inició en 1987 con la publicación de México Profundo.

Los movimientos ciudadanos, son nuevas formas de expresión de las identidades colectivas como una respuesta a las reformas del libre mercado de los noventas y son diferentes desde otras formas de movilización social, tales como los retos populistas e insurgentes al orden social. Los movimientos ciudadanos son nuevas formas de acción colectiva y movimientos de protesta que emergen en los espacios de la nueva sociedad civil en Latinoamérica. La contradicción entre las crecientes presiones en las instituciones financieras internacionales y el estado ampliado, con la consolidación en los noventas del movimiento ambientalista transnacional y los derechos de las comunidades indígenas para sostener sus propios proyectos de vida, fueron legitimados y los movimientos indígenas y ambientales consolidados.

Los nuevos movimientos sociales indígenas en Latinoamérica y el Caribe tienen un impulso en 1992, hasta que solamente tuvo un alcance nacional y local. Varios hechos han elevado la conciencia de los nuevos movimientos indígenas. Los movimientos indígenas americanos iniciaron en 1992 con la legitimación de los asuntos indígenas y la conciencia internacional, que vino del énfasis del 500 aniversario del descubrimiento de las Américas.

El año de 1992 fue un tiempo de renovación para los movimientos indígenas, cuando Rigoberta Menchu, una mujer Maya fue galardonada con el Premio Nobel de la Paz en 1992, por su papel como símbolo para elevar su conciencia de los nuevos movimientos indígenas. La celebración vitaliza a las organizaciones y a las conexiones de movimientos indígenas, creando nuevas alianzas entre los grupos indígenas, los movimientos para indígenas, Organizaciones No Gubernamentales, grupos ambientales y otros. Esto y otros eventos crearon un ambiente en México para la formación de nuevas organizaciones de movimientos indígenas y nuevas alianzas entre los movimientos indígenas.

Un movimiento social indígena y religioso, la sociedad civil Las Abejas, emergió en 1992 como una coalición de comunidades locales indígenas Maya-Tzotzil, quienes se unieron para solucionar conflictos de la tierra. El movimiento de Las Abejas es una expresión de la sociedad civil que comprime diversas organizaciones de campesinos, indígenas y del gobierno local. Desde 1997, cuando 45 miembros fueron masacrados en Acteal, organizaciones de derechos humanos apoyan los usos no violentos de métodos de resolución de conflictos. Las interacciones entre miembros, de una única identidad sincrética global, la cual inspira el movimiento en su acción colectiva de resistencia contra el desplazamiento e invasión de tierras. 
El Congreso Nacional Indígena empezó en 1996 a traer consigo los movimientos indígenas alineados con los Zapatistas, bajo el supuesto de crear un entendimiento del sentido colectivo de sí mismo en movimiento, en contraste a homogeneizar el mestizaje y las categorías de identidad, así como a ganar reconocimiento como ciudadanos.

La reforma neoliberal en México, ha servido como crisol por la emergencia de nuevos actores entre los movimientos indígenas tales como los Zapatistas. La auto organización de los campesinos y la simpatía del movimiento de los Zapatistas prosperó en los finales de los ochentas y los principios de los noventas en las comunidades de campesinos que buscaban tierras. Los Zapatistas se han posicionado por sí mismos en diálogo con los movimientos sociales anti neoliberales en México y alrededor del mundo. El EZLN ofrece una alternativa coherente a los movimientos sociales en México.

La emergencia de los nuevos movimientos sociales como centro de oposición al TLCAN, son el resultado de movimientos tales como El Barzón y los Zapatistas (EZLN). El Ejército Zapatista de Liberación Nacional (EZLN) es un nuevo tipo de movimiento de guerrilla que por cualquier coincidencia, el día que escogieron para lanzar su movimiento, el primero de enero de1994, fue también el día que el TLCAN entró en vigor.

Como un movimiento social, el EZLN se distingue de otros movimientos populares en México, porque han movilizado activamente no solamente a los grupos indígenas sino también otros grupos grandes de actores económicos, sociales y políticos. Las comunidades indígenas re-enfocan en los valores tradicionales y transforman los viejos significados a fin de continuar con sus luchas de tierras. El EZLN puede estratégicamente desarrollarse en un nuevo movimiento político o en un movimiento autónomo indígena.

En enero del 94, la revuelta Zapatista de las comunidades indígenas Mayas en Chiapas recibió solidaridad de las organizaciones de los movimientos indígenas y de campesinos, redes, alianzas y coaliciones. El movimiento de la guerrilla Zapatista que surge en Chiapas en 1994 ha disparado los movimientos sociales en México y en el extranjero, para enfatizar el incremento de los niveles de pobreza bajo las políticas económicas neoliberales y demandar una más equitativa distribución de ingreso. Como movimiento de resistencia, la revuelta Zapatista en Chiapas ha sido exitosa usando el slogan ¡Basta ya! y presentando su programa de once palabras al gobierno mexicano: "Trabajo, Tierra, Techo, Pan, Salud, Educación, Democracia, Libertad, Paz, Independencia y Justicia”.

El movimiento Zapatista representa a las organizaciones de base, las cuales pueden ampliar y diversificar sin las alianzas externas. En 1994, diversos movimientos cívicos locales, que incluyeron a los movimientos de los derechos humanos, cooperativas y derechos étnicos, emergieron a través de la región en conflicto en Chiapas sacando la mayoría de los presidentes municipales del partido gobernante e instalando consejos de pueblos plurales. La estrategia diseñada para perseguir sus demandas y relaciones administrativas con las instituciones del Estado, son dos factores de los movimientos indígenas para ser exitosos. 
Un movimiento de solidaridad emanando de las organizaciones sociales para apoyar al EZLN creció en proporciones masivas. El día del nuevo año de 1994, marca el inicio del TLCAN y el arribo de un nuevo movimiento de guerrilla identificado con Emiliano Zapata, el héroe agrario, símbolo de la liberación nacional y de la resistencia de las gentes indígenas de México, desplazadas de las grandes tenencias de la tierra.

Los éxitos sin precedentes del EZLN como un nuevo movimiento social ha sido atribuido a su post modernidad. El EZLN como un nuevo movimiento social ha estado caracterizado como la primer "potsmoderna" rebelión por las técnicas de comunicación sofisticadas empleadas. Un analista mexicano, Gustavo Esteva, cuestionó si era la última guerrilla de América Central o se ha iniciado la nueva era de la revolución postmoderna.

El movimiento Zapatista es más grande que el Ejército Zapatista de Liberación Nacional (EZLN) porque involucra a numerosos movimientos indígenas y de campesinos, y organizaciones civiles. Zapata ha inspirado los movimientos en México y su lucha por sostener y mejorar las formas de vida. Una red de apoyo al movimiento Zapatista fue iniciado por otros movimientos sociales.

El movimiento Zapatista parece llenar la definición de un nuevo movimiento social porque concierne con la identidad étnica, busca su total autonomía de las organizaciones y los partidos políticos, llama por una liberación cultural y sobrevivencia de los indígenas de México y de los campesinos de Chiapas, el consumo colectivo y la demanda de servicios públicos. El movimiento de los Zapatistas en México ha retado el status quo de las estructuras económicas internacionales, bajo los procesos de globalización económica.

El movimiento Zapatista busca la autonomía indígena dentro del marco de referencia de la nación mexicana. Los activistas locales del movimiento Zapatista han empujado el conflicto a la arena internacional movilizando movimientos transnacionales contra el Estado nación y debilitado los esfuerzos de los gobiernos locales para ocultar la naturaleza del conflicto. Los movimientos indígenas como los Zapatistas han rechazado la subordinación a los partidos políticos, luchando por la autodefinición y las prácticas culturales. El Zapatismo administra los tiempos poniendo a las comunidades primero, distanciando los movimientos de los eventos nacionales.

La teología de la liberación trabaja para liberar a la gente pobre y oprimida en nuestro mundo. No obstante, los movimientos de la teología de la liberación en Latinoamérica han declinado y no reconocen su nueva expresión en las teologías indígenas.

El Gobernador Madrazo en el Estado de Tabasco en 1994, enfrentó demandas de los movimientos populares de campesinos e indígenas, quienes fueron dañados por la industria petrolera. La situación llevó a un claro deterioro de la actividad económica de la población, generando protestas expresadas a través de los movimientos sociales tales como el "Pacto Ribereño". Cuando más de un centenar de participantes fueron arrestados, Global Exchange y cuatro organizaciones no gubernamentales enviaron representantes a Tabasco. 
Un número creciente de Organizaciones No Gubernamentales y movimientos sociales en México y Centroamérica ha elevado sus preocupaciones acerca del Plan Puebla Panamá, como una expresión de la agenda de integración económica más amplia en la región de Centroamérica, que abarca de Puebla a Panamá, comprendiendo los siete países de Centroamérica así como los Estados del Sur de México.

Muchas comunidades indígenas, movimientos sociales y Organizaciones No Gubernamentales han condenado el lavado y destrucción de la naturaleza verde del plan masivo del Plan Puebla Panamá. Hay un movimiento más grande que se opone, al impuesto desde arriba, Plan Puebla Panamá que podría guiar a la estrategia más inclusiva de desarrollo alternativo.

Los movimientos sociales indígenas contemporáneos han estado evolucionando en organizaciones en redes capaces de desatar "netwars sociales transnacionales", como redes transnacionales emergentes de activismo de la era de información basadas en asociaciones entre organizaciones no gubernamentales (ONGs), relacionadas con asuntos modernos y postmodernos tales como el medio ambiente, los derechos humanos, inmigración, gentes indígenas y libertad en el espacio (Fukuyama, 1998). Sin embargo, los actores colectivos se dispersan, fragmentan y atomizan en redes las cuales rápidamente desaparecen de la relevancia política en sectas, círculos de apoyo emocional y grupos de terapia.

\section{La ecología política indígena}

Carruthers (1995) usa el término ecología política indígena para referirse a la consolidación de las alianzas de los movimientos sociales basados en un esfuerzo para preservar, defender, aplicar e integrar el conocimiento de la ecología tradicional incorporada en la cultura indígena campesina y prácticas en esfuerzos de desarrollo de base. La ecología indígena se refiere a las alianzas entre las organizaciones de movimientos ambientales e indígenas. La internacionalización del movimiento de la ecología indígena revela una tensión entre dos visiones de movimientos sociales contendientes, una de las cuales es la propia actividad del movimiento social.

Los analistas de los movimientos sociales sobre miran la práctica de la ecología política indígena y la acción de los movimientos sociales, que crean espacios políticos significativos, como un puente de negociación entre los movimientos sociales y el Estado, para asegurar los beneficios y para alentar la capacidad social de las organizaciones de movimientos sociales contra el Estado.

Los movimientos sociales ecológicos son intrínsecamente multi sectoriales, fomentan las ligas entre los movimientos de indígenas, campesinos, salud pública, laborales, estudiantes y urbano popular. Los movimientos socio-ecológicos articulan preocupaciones y asuntos de los movimientos indígenas y de otros movimientos sociales. 
Se han realizado fuertes debates dentro de los movimientos ambientalistas sobre lo que debe ser entendido como desarrollo sustentable y una falta relativa de investigación en los movimientos contemporáneos, para proteger el ambiente iniciado por actores no indígenas. Los movimientos indígenas tienen recursos y medios a las normas ambiguas acerca de los derechos humanos, la sustentabilidad ambiental y la diversidad cultural.

El ambiente mexicano y los movimientos indígenas se enfocan en la agricultura y en el cuestionamiento por las alternativas sustentables, la lucha por revalidar, revitalizar e integrar el conocimiento ecológico tradicional en proyectos de desarrollo de base. La agroecología indígena, como un movimiento social, busca rescatar las prácticas tradicionales, para proteger la diversidad cultural y biológica y para traer juntos formas ambientales del Primer Mundo y el Tercer Mundo. El emergente movimiento agroecológico en México está apresurado por los altos costos de la industria agrícola.

La experimentación agroecológica está alentada por agencias de Estado que mantienen conexiones con activistas en los movimientos de campesinos y ambientalistas. La resistencia a los movimientos contra la agricultura y la tecnología corporativa ha emergido. Hay movimientos nacionales de la resistencia contra el uso de recursos ambientales y servicios.

La preservación de diversidad de especies es intrínseca a la sustentabilidad del desarrollo y a los movimientos ambientales y el movimiento internacional de derechos indígenas. Los movimientos agrarios y étnicos son conscientes de la lucha por la identidad y empiezan a establecer sus demandas como derechos, derechos de las tierras, o derechos educacionales. Los movimientos indígenas necesitan seguir una estrategia de múltiples direcciones de cabildeo, hacer alianzas, apelar a las cortes y campañas públicas, que una alternativa legal, la cual es asediada por trampas y resultados contra productivos.

La lucha de los pueblos indígenas tarahumaras de Chihuahua, es articulado contra las prácticas forestales ilegales, en particular, el agua y la biodiversidad. La red de Acción de Investigación de la Tierra fue lanzada en Chiapas para movilizar la gente en apoyo de los movimientos populares quienes están luchando por acceso a la tierra. La identidad entre las comunidades provee involucramiento emocional en la acción colectiva.

Los movimientos sociales indígenas sostenidos en México y la gente indígena tienen un importante rol para lograr resoluciones y demandas del Congreso Indígena Nacional y los movimientos sociales en México dirigidos a proteger y conservar la biodiversidad natural y cultural. Un movimiento ambiental en el Sureste de México y Centroamérica pretende preservar la salud de la biodiversidad.

\section{La transnacionalizacion de los movimientos indígenas}

Complejas redes transnacionales a través de las fronteras entre los Estados, mercados y la sociedad civil, son medios de comunicación de movimientos de las gentes indígenas, 
ambientalistas y de derechos humanos. Un movimiento poco conocido puede proyectar sus reclamaciones identificándose a sí mismo como movimiento en contra de las corporaciones transnacionales, echándole la culpa al villano común para forjar ligas entre movimientos distantes. Los movimientos pueden tratar de conectar por sí mismo a un medio externo cuando el conocimiento local, es escaso.

El concepto de redes es para analizar las relaciones sociales, las interacciones grupales, la conducta organizacional, los movimientos sociales y los movimientos transnacionales (Mitchel, 1969). La capacidad de la red de los movimientos ha sido estudiada en la fortaleza de las organizaciones indígenas, en la habilidad para crear ligas débiles, creación de redes de comunicación entre los movimientos y las comunidades en ambos niveles, local y global. Los movimientos sociales indígenas hacia una red de bienestar están ampliando los patrones de las relaciones políticas. Un nuevo aspecto de estos movimientos es su articulación regional y su participación en las redes y manifestaciones en movimientos anti globalización.

Los movimientos indígenas de base interactúan con los aliados internacionales gracias a los avances tecnológicos en las comunicaciones. La internacionalización de la sociedad civil se refiere a las ligas transfronterizas establecidas por las organizaciones de los movimientos sociales de paz, derechos humanos, ambientales, género y trabajo, indígenas y otros movimientos. La internacionalización de los movimientos sociales es una respuesta espontánea a proteger y defender la gente vulnerable y la estrategia para alentar su capacidad y para obtener espacio autónomo independiente del Estado.

La internacionalización de los movimientos sociales indígenas de México encuentra apoyo alrededor del mundo. El movimiento Zapatista es considerado un rizoma transnacional o una red de guerras social que usa la ola de fábrica electrónica global de la lucha y monta una red de guerra social contra un Estado que se retraza en la democratización. La rebelión Zapatista es un prototipo de una red de guerras social en el Siglo 21, en el cual casi cada aspecto de las comunicaciones modernas por computadora ha sido usado.

Hay una nueva capacidad para esto y otros movimientos sociales para comunicarse a través de las fronteras y para operar en el nivel transnacional. Los análisis de éste movimiento reconocen como el contenido de los rizomas o formas de movilización social en red, jalan juntos a los movimientos de base contra el orden económico y político actual de México y del mundo y facilitan los acercamientos alternativos a la organización social. Los gobiernos deberían aprender a actuar en contra de tales movimientos sociales y empezar a mejorar su habilidad para manejar la contra guerra de las redes.

Hay otros movimientos binacionales tales como el Frente Indígena Oaxaqueño Binacional compuesto de organizaciones de trabajadores emigrantes indígenas con base en Estados Unidos, incluyendo el Comité Cívico Popular Mixteco, la organización regional Oaxaqueña, La Comunidad Tlacolulense en Los Ángeles, la Organización de Gentes Explotadas y Oprimidas, etc., y mantienen las afiliaciones con los Trabajadores del Campo Unidos de América César Chávez y el Grupo de Trabajo de Tecnología 
Apropiada del Instituto Herat Island, una Organización No Gubernamental con base en San Francisco, California.

El Frente Mixteca-Zapoteca Binacional con base en California, organiza a los trabajadores emigrantes originaros de Oaxaca, promueve la resistencia indígena en defensa de los derechos humanos y de los trabajadores, y el desarrollo sustentable de proyectos de la agricultura.

La internacionalización de las ligas de los movimientos y la solidaridad transfronteriza son medios para negociar cambios locales con el Estado para asegurar los beneficios. Los movimientos indígenas de México toman su caso por una nueva ley indígena para la Organización Internacional del Trabajo.

Los reclamos de los movimientos de los derechos humanos han dado lugar a la noción de una comunidad global. La globalización ha jugado una más activa participación en los movimientos indígenas en el incremento de la sociedad civil global. Una estrategia multi direccional incluye alianzas con otros movimientos sociales dependientes en la existencia de puntos de interés común.

Carruthers (1996) analiza la formación de alianzas entre movimientos contemporáneos, los grupos ambientales y los grupos urbanos formados por las clases medias educadas que han encontrado una convergencia de intereses con las organizaciones indígenas y de campesinos existentes del segmento más marginal del pueblo rural de México. Las comunidades indígenas aliadas con los movimientos ambientales y de derechos humanos pueden cabildear y ejercitar presiones en los gobiernos nacionales. La alianza de los movimientos indígenas y otros movimientos sociales pueden tener algunas posibilidades de rehacer las estructuras actuales de gobernabilidad.

Las alianzas de movimientos sociales, ambientales y agroecológicos indígenas, promueve y fortalece las conexiones entre las organizaciones de base (OB), que representan las organizaciones indígenas, de campesinos, y pequeños propietarios existentes, y organizaciones de base de apoyo, las cuales representan la fase aplicada de los movimientos ambientales y agroecológicos. Una organización de base de apoyo es una entidad cívica de desarrollo que provee servicios y canaliza recursos a los grupos locales de familias e individuos urbanos o rurales en desventaja.

Reuniones entre los movimientos y en gran escala, son encarnados en el movimiento Zapatista. Las comunidades Zapatistas son islas de liberación relativa para las mujeres y son una importante fuente de los atractivos del movimiento. La participación de las mujeres indígenas en los movimientos desde 1994, una década de prácticas políticas de los movimientos, apoya el argumento de la multi dimesionalidad de la identidad y las experiencias de opresión por el Estado mexicano para crear las dicotomías entre los derechos de las mujeres y los derechos de los indígenas.

Las mujeres emergen en el liderazgo de muchos de los nuevos movimientos en colaboración con los activistas y feministas del movimiento estudiantil anterior. Una Ley 
Revolucionaria de las Mujeres en el movimiento Zapatista atrae la atención de los activistas en las redes de las mujeres en el ciberespacio. Estas redes tienen un rol activo en circular información acerca de las mujeres indígenas que participan en el movimiento Zapatista.

Por ejemplo, los movimientos sociales de los derechos de las mujeres han llevado a cabo una extensiva red binacional para el aprendizaje e intercambio mutuo para apoyar las bases de formación de poder de las mujeres trabajadoras y las mujeres indígenas. Las organizaciones de mujeres canadienses construyen solidaridad a través de las fronteras porque las mujeres de color tienen una fuerte voz en el movimiento feminista canadiense. Otro buen ejemplo, son las Organizaciones No gubernamentales de apoyo y defensa son jugadores en la frontera en las áreas de amenazas ambientales, los movimientos de justicia ambiental, derechos humanos, derechos indígenas, entre otros.

\section{CONCLUSIÓN}

Las comunidades indígenas están cohesivamente respondiendo a los conflictos étnicos, como uno de los movimientos sociales más significativos en la historia de México para la sorpresa de otros actores sociales, tales como el gobierno, los políticos, analistas y académicos.

Sin embargo, el componente étnico de los movimientos indígenas se orienta a la conclusión que no pueden ser estudiados como otros movimientos sociales bajo un acercamiento teórico de los movimientos sociales.

Los movimientos sociales indígenas son medios de desarrollo de la identidad social y recuperación democrática, mientras que organizan las movilización política contra la dictadura. A través del uso creativo de imágenes e información, la gente indígena ha volteado la marginalidad y la pobreza en su más grande fortaleza en la emergencia de los movimientos basados en la identidad internacionalizada.

Nelly (2002), establece que la forma en que estamos oprimidos o la mera crónica de las locuras y estupideces de movimientos radicales no parecen muy útiles. Los movimientos sociales progresivos no simplemente producen estadísticas y narrativas de opresión, también los mejores hacen lo que la poesía logra, transportarnos a otro lugar, obligarnos a aliviar horrores y más importantes, nos permite imaginarnos una nueva sociedad.

Los movimientos indígenas con frecuencia apoyan metas de partidos en retorno de beneficios, pero la participación directa puede atrapar movimientos en la oposición intransigente. Los gobiernos no han desmovilizado a los movimientos populares, pero han causado nuevas divisiones de movimientos sociales co-optados tales como los Piqueteros en Argentina y los movimientos indígenas en Bolivia y Ecuador. Sin embargo los movimientos de los sin tierra de Brasil eluden ambas posiciones (Zibechi, 2004). 
La emergencias de los gobiernos de centro izquierda en Latinoamérica, apoyados por movimientos indígenas de una amplia base social debilitan y causan crisis en el modelo neoliberal prevaleciente $\mathrm{y}$ enfrentan una amplia gama de nuevos dilemas $\mathrm{y}$ cuestionamientos. Los partidos de la izquierda apoyan a los movimientos indígenas y populares, dan incentivos selectivos como candidaturas y posiciones de liderazgo, etc., para atraer movimientos populares. El liderazgo de movimientos con frecuencia gana experiencia política lejos de casa y trae aliados.

Los líderes de los movimientos indígenas en tales posiciones pueden alentar el prestigio de su propio movimiento, mantener informado a sus apoyos y mantener ligas cercanas con varios líderes. En la consolidación desde debajo de la sociedad civil en las regiones indígenas, los aliados externos pueden ser cruciales a la capacidad de los movimientos para sobrevivir (Harvey, 1994; Hernández, 1994).

La revuelta de Chiapas permanece como una revuelta indígena, porque es una revuelta del EZLN apoyados con el resto de los movimientos sociales en México. Es todavía temprano para evaluar el grado del movimiento del EZLN que está logrando cambiar de cultura política a través de elementos que son parte de la historia de los movimientos, tales como la democracia radical y las prácticas autónomas, claman su identidad indígena, tradiciones y dignidad. Los movimientos indígenas mantienen algunos niveles de autonomía y ha incrementado su capacidad de negociar con el Estado.

La participación de los movimientos indígenas en los movimientos de democratización adoptan formas organizacionales para establecer relaciones de control y entradas con los procesos políticos y judiciales que les afectan de tal forma que pueden extender sus quejas a través de los partidos políticos, Organizaciones No Gubernamentales, etc., con acceso directo a los procesos de tomas de decisiones en los diferentes niveles de gobierno. La orientación estratégica hacia adentro de los movimientos indígenas hacia el contexto nacional depende del nivel del control y entrada. El peso demográfico de los movimientos indígenas es nacional y regionalmente importante.

\section{LITERATURA CITADA}

Boal, F. W. y J. Neville H.D.

1982 "Integration and Division: Geographical Perspectives on the Northern Ireland Problem. London.” Academic Press. 86 p.

Brecher, J. y T. Costello.

1994 "Global Village or Global Pillage? Economic, Reconstruction from the Bottom Up”. South End Press. 120 p. 
Cardoso, R. y L. Correa

1987 "Movimientos Sociais na América Latina", 1(3): 34-78.

Caruthers, D. V.

1996 "Indigenous ecology and the politics of linkage in Mexican social movements". Third World Quarterly 17 (5):1007-1028

Carruthers, D.

1995 "The Political Ecology of Indigenous Mexico: Social Mobilization and State Reform." Ph.D. diss., University of Oregon, Eugene, Oregon, USA. 232 p.

Connor, W.

1977 "Ethnonationalism in the First World: The Present in Historical Perspective". In: Milton J. Esman (ed.). $144 \mathrm{p}$

Curtin, Ch.; Kelly, M. y Liam O’D.

1984 “Culture and Ideology in Ireland”. Galway: Officina Typograhica Galway University Press. Officina Typograhica Galway University Press. 145 p.

Deutsch, K.

1963 "Nerves of Government: Models of Political Communication and Control". New York: The Free Press. 147 p.

Deutsch, K.

1962 "Nationalism and Social Communication: An Inquiry into the Foundations of Nationality". Cambridge, Ma.: The M.I.T. Press

Evers, T.

1985 "Identity: The Hidden Side of New Social Movements in Latin America," David Slater, ed., New Social Movements and the State in Latin America.Amsterdam: CEDLA. 178 p.

Fukuyama, F.

1998 "Review of Activists Beyond Borders: Advocacy Networks in International Politics, in Foreign Affairs". 77(4): July-August 123 p.

1994 "Comments on Nationalism and Democracy". In: Larry Diamond \& Marc F. Plattner (eds). $345 \mathrm{p}$. 
Harvey, N.

1994 "Rebellion in Chiapas: Rural Reforms, Campesino Radicalism and the Limits to Salinismo". Transformation of Rural Mexico, no. 5. La Jolla: University of California, Center for U.S.-Mexican Studies. 32 p.

Havel, V.

1985 "The Power of the Powerless". In: John Keane (ed.) The Power of the Powerless: Citizens Against the State in Central-Eastern Europe. London:Hutchinson. 244 p.

Hernández, N.L

1994 "The Chiapas Uprising." Transformation of Rural Mexico, no. 5. La Jolla: University of California, Center for U.S.-Mexican $\backslash$ Studies. 53 p.

Hroch, M.

1996 Nationalism and National Movements: Comparing the Past and the Present of Central and Eastern Europe" Nations and Nationalism. Journal for the Association for the Study of Ethnicity and Nationalism 2(1): 35-44.

Maguire, D.

1996 Irish Republicans since 1968: From Rebels to Reformers? Sydney: University of Sydney. 98 p.

Mejía P. M. C. y Sergio S. S.

1987 La lucha indígena: Un reto a la ortodoxia. IIS/Siglo XXI. México D. F. 54 p.

Moguel, J.; Botey, C. y Hernández, L.

1992 "Autonomía y nuevos sujetos sociales en el desarrollo rural". Siglo XXI/CEHAM. México, D.F. 75 p.

Nairn, T.

1993 "Internationalism and the Second Coming" Daedalus: Journal of American Academy of Arts and Sciences. Reconstructing Nations and States 122: 155-170.

Schöpflin, G.

1995 "Nationalism and Ethnicity in Europe, East and West" In: Charles A. Kupchan (ed.) Reconstructing Nations and States 122: 155-170

Tarrow, S.

1996 "States and Opportunities: The Political Structuring of Social Movements". In: Doug McAdam; John D. McCarthy \& Mayer N. Zald (eds) Reconstructing Nations and States 122: 155-170. 
Tilly, C.

1993 “National Self-Determination as a Problem for All of Us". Daedalus: Journal of American Academy of Arts and Sciences. Reconstructing Nations and States 122: 29-36.

Warman, A.y Argueta, A.

1993 “Movimientos indígenas contemporáneos”. CIIH/UNAM/Miguel Ángel Porrúa Mexico, D.F. 85 p.

Watson, C.

1996 "Folklore and Basque Nationalism: Language, Myth, Reality" Nations and Nationalism: Journal for the Association for the Study of Ethnicity and Nationalism 2(1): 17-34pp.

Wilson, D. y Jerry T.

1996 “Institutions for Conciliation and Mediation" In: Seamus Dunn (ed.). Journal for the Association for the Study of Ethnicity and Nationalism 2(1): 35-42pp.

Zibechi, R.

2004 “A Panorama of Social Movements in South America”. Dangerous. Liaisons: Center-Left Governments \& the Grassroots. Americas Program, Interhemispheric Resource Center (IRC) December 7. 